\title{
A covariant approach for perturbations of rotationally symmetric spacetimes
}

\author{
Chris Clarkson \\ Cosmology and Gravity Group, Department of Mathematics and Applied Mathematics, \\ University of Cape Town, Rondebosch 7701, Cape Town, South Africd*
}

(Dated: November 2, 2018)

\begin{abstract}
We present a covariant decomposition of Einstein's Field Equations which is particularly suitable for perturbations of spherically symmetric - and general locally rotationally symmetric - spacetimes. Based upon the utility of the $1+3$ covariant approach to perturbation theory in cosmology, the semitetrad, $1+1+2$ approach presented here should be useful for analysing perturbations of a variety of systems in a covariant and gauge-invariant manner. Such applications range from stellar objects to cosmological models such as the spherically symmetric Lemaître-Tolman-Bondi solutions or the class of locally rotationally symmetric Bianchi models.
\end{abstract}

\section{INTRODUCTION}

Tetrad formalisms in general relativity have played a pivotal role in its development as well as our understanding of the subject. These range from the complex null tetrad of Newmann and Penrose, to the $1+3$ approach of Ehlers, Ellis and others, which includes both a full tetrad approach as well as a partial 'covariant' approach where only one timelike tetrad vector is chosen (see [1] for a review and references). These techniques formulate the equations of general relativity as first-order differential equations in the physical curvature and dynamic variables of the covariant derivatives of the tetrad vectors, as opposed to the more usual coordinate approach involving second-order partial differential equations in functions appearing in the metric. The differential operators which appear are convective derivatives along the tetrad vectors as opposed partial derivatives with respect to particular coordinates. Much of their utility arises in spacetimes with special symmetry. For example, the $1+3$ covariant approach is perfect for cosmology because it covariantly factorises out the essential coordinate - time - leaving all the background field equations as covariant scalar equations. Under perturbations all 3-vectors and tensors (which must vanish in the background due to homogeneity and isotropy) become gauge-invariant first-order quantities making a Fourier analysis easy [1, 2].

We formulate here an approach which involves a semi-tetrad: we keep the timelike threading vector field of the $1+3$ approach and introduce one spatial vector. The remaining two dimensions are left untouched, rather like the ' 3 ' in the $1+3$ approach. Indeed the formalism presented here may be considered as half-way between the $1+3$ tetrad and covariant approaches. A similar approach has been discussed before in [3, 4, [5, [6], and we expand on this considerably here by presenting the full system of $1+1+2$ equations.

It is expected that this approach may find use in perturbations of spacetimes with a preferred spatial direction at each point - so-called locally rotationally symmetric spacetimes 7]. These include the spherically symmetric Lemaître-Tolman-Bondi models, many classes of Bianchi models, as well as forming the background for most stellar models. In this paper we provide the algorithm of how to calculate gravitational perturbations in any LRS spacetime in a covariant and gauge-invariant (GI) way.

As an example of it's utility, such a covariant perturbative scheme was applied to the Schwarzschild solution in [8]. Despite being a well understood problem, it was shown using the $1+1+2$ approach how both the axial and polar degrees of freedom may be unified into a single transverse traceless tensor which obeys the tensorial form of the Regge-Wheeler equation [8, 9]

$$
-\ddot{W}_{a b}+\hat{\hat{W}}_{a b}+\mathcal{A} \hat{W}_{a b}-\phi^{2} W_{a b}+\delta^{2} W_{a b}=0,
$$

where the Regge-Wheeler tensor $W_{a b}$ is a gauge- and frame-invariant TT tensor, defined in [8] (other variables are defined below), and ', ${ }^{\prime}$ and $\delta$ are time, radial and angular derivatives, respectively. This tensor contains in compact form the curved space generalisation of the two flat space GW polarizations $h_{+}$and $h_{\times}$[10] (see also [11] for an extension of this work). The approach here also been used to study scalar and electromagnetic perturbations of LRS spacetimes, and generalised Regge-Wheeler equations were found [12, 13]. Furthermore, it has been used to study the interaction of magnetic fields and gravitational waves around a black hole - a process which produces electromagnetic radiation mirroring the gravitational waves [10].

*Electronic address: chris.clarkson@uct.ac.za 
In Sec. II we discuss the $1+1+2$ approach in full generality, and then in Sec. III we discuss the perturbation procedure for LRS spacetimes, before sumarising in Sec. IV.

\section{FORMALISM}

In the $1+3$ approach, a timelike threading vector field $u^{a}\left(u^{a} u_{a}=-1\right)$ is introduced, representing the observers' congruence. Given this vector field, the projection tensor $h_{a}^{b}=g_{a}^{b}+u_{a} u^{b}$ is introduced, which projects all vectors and tensors orthogonal to $u^{a}$. Using $h_{a b}$, any 4 -vector may be split into a $\left(1+3\right.$ scalar) part parallel to $u^{a}$ and a (3-vector) part orthogonal to $u^{a}$. Any second rank tensor may be covariantly and irreducibly split into scalar, vector andprojected, symmetric, trace-free (PSTF) 3-tensor parts, which requires the alternating tensor $\varepsilon_{a b c}=u^{d} \eta_{d a b c}[1]$. Tensors of higher rank may be similarly split, but are rarely used (an important exception being cosmic microwave background physics [14, 15]). These are the fundamental quantities describing the spacetime, after the introduction of $u^{a}$.

We now introduce another vector field and perform another split, but this time of the $1+3$ equations. The ' $1+1+2$ ' decomposition we develop here has been partially studied before, mostly in the context of symmetries of solutions of the EFE [5, 16]. It was introduced by [3] and further developed in [4, 8, 11, 12, 13]. However, there are importances differences with the work presented here. In the following we assume the $1+3$ covariant split of the equations (as given in [1], for example), with all tensors split into scalars, vectors and PSTF tensors with respect to $u^{a}$.

Take a unit vector $n^{a}$ orthogonal to $u^{a}: n^{a} n_{a}=1, u^{a} n_{a}=0$, and define the projection tensor

$$
N_{a}^{b} \equiv h_{a}^{b}-n_{a} n^{b}=g_{a}^{b}+u_{a} u^{b}-n_{a} n^{b},
$$

which projects vectors orthogonal to $n^{a}$ (and $\left.u^{a}\right): n^{a} N_{a b}=0=u^{a} N_{a b}$, onto 2-surfaces $\left(N_{a}{ }^{a}=2\right)$ which we refer to as the sheet. This is also the screen space of the null vector $k^{a} \propto u^{a}+n^{a}$.

Any 3 -vector $\psi^{a}$ can now be irreducibly split into a scalar, $\Psi$, which is the part of the vector parallel to $n^{a}$, and a vector, $\Psi^{a}$, lying in the sheet orthogonal to $n^{a}$;

$$
\psi^{a}=\Psi n^{a}+\Psi^{a}, \quad \text { where } \Psi \equiv \psi_{a} n^{a}, \quad \text { and } \quad \Psi^{a} \equiv N^{a b} \psi_{b} \equiv \psi^{\bar{a}},
$$

where we use a bar over an index to denote projection with $N_{a b}$ on that index. Similarly, any PSTF tensor, $\psi_{a b}$, can now be split into scalar, vector and tensor (which are PSTF with respect to $n^{a}$ ) parts:

$$
\psi_{a b}=\psi_{\langle a b\rangle}=\Psi\left(n_{a} n_{b}-\frac{1}{2} N_{a b}\right)+2 \Psi_{(a} n_{b)}+\Psi_{a b},
$$

where

$$
\begin{aligned}
\Psi & \equiv n^{a} n^{b} \psi_{a b}=-N^{a b} \psi_{a b} \\
\Psi_{a} & \equiv N_{a}{ }^{b} n^{c} \psi_{b c}=\Psi_{\bar{a}} \\
\Psi_{a b} & \equiv \psi_{\{a b\}} \equiv\left(N_{(a}{ }^{c} N_{b)}{ }^{d}-\frac{1}{2} N_{a b} N^{c d}\right) \psi_{c d} .
\end{aligned}
$$

We use curly brackets to denote the PSTF with respect to $n^{a}$ part of a tensor. Note that for 2 nd-rank tensors in the $1+1+2$ formalism 'PSTF' is precisely equivalent to 'transverse-traceless'. Note also that $h_{\{a b\}}=0, N_{\langle a b\rangle}=-n_{\langle a} n_{b\rangle}=$ $N_{a b}-\frac{2}{3} h_{a b}$.

We also define the alternating Levi-Civita 2-tensor

$$
\varepsilon_{a b} \equiv \varepsilon_{a b c} n^{c}=u^{d} \eta_{d a b c} n^{c},
$$

so that $\varepsilon_{a b} n^{b}=0=\varepsilon_{(a b)}$, and

$$
\begin{aligned}
\varepsilon_{a b c} & =n_{a} \varepsilon_{b c}-n_{b} \varepsilon_{a c}+n_{c} \varepsilon_{a b} \\
\varepsilon_{a b} \varepsilon^{c d} & =N_{a}{ }^{c} N_{b}{ }^{d}-N_{a}{ }^{d} N_{b}{ }^{c} \\
\varepsilon_{a}{ }^{c} \varepsilon_{b c} & =N_{a b}, \quad \varepsilon^{a b} \varepsilon_{a b}=2 .
\end{aligned}
$$

Note that for a 2-vector $\Psi^{a}, \varepsilon_{a b}$ may be used to form a vector orthogonal to $\Psi^{a}$ but of the same length.

With these definitions we may split any object into scalars, 2-vectors in the sheet, and transverse-traceless 2tensors, also defined in the sheet. These three types of objects are the only objects which appear, after a complete decomposition. Hereafter, we will assume such a split has been made, and 'vector' will generally refer to a vector projected orthogonal to $u^{a}$ and $n^{a}$, and 'tensor' will generally mean transverse-traceless tensor, defined by Eq. (5). 
There are two new derivatives of interest now, which $n^{a}$ defines, for any object $\psi_{\ldots}{ }^{\cdots}{ }^{\cdots}$ :

$$
\begin{aligned}
\hat{\psi}_{a \cdots b}{ }^{c \cdots d} & \equiv n^{e} \mathrm{D}_{e} \psi_{a \cdots b}{ }^{c \cdots d}, \\
\delta_{e} \psi_{a \cdots b}{ }^{c \cdots d} & \equiv N_{e}{ }^{j} N_{a}{ }^{f} \cdots N_{b}{ }^{g} N_{h}{ }^{c} \cdots N_{i}{ }^{d} \mathrm{D}_{j} \psi_{f \cdots g}{ }^{h \cdots i} .
\end{aligned}
$$

The hat-derivative is the derivative along the vector field $n^{a}$ in the surfaces orthogonal to $u^{a}$. This definition represents a conceptual divergence from $1+3$ tetrad approach, in which the basis vectors appear on an equal footing [i.e., with $\nabla_{a}$ rather than $\mathrm{D}_{a}$ in Eq. (10)]. As a result, the congruence $u^{a}$ retains the primary importance it has in the $1+3$ covariant approach. (We choose to think of $\mathcal{A} \equiv u^{a} n^{b} \nabla_{a} u_{b}=-u^{a} u^{b} \nabla_{a} n_{b}$ as the radial component of the acceleration of $u^{a}$, rather than the time component of $\dot{n}^{a}$.) The $\delta$-derivative, defined by Eq. (11) is a projected derivative on the sheet, with projection on every free index.

These derivatives then affect our projection tensor $N_{a b}$ and Levi-Civita tensor as follows:

$$
\begin{aligned}
\dot{N}_{a b} & =2 u_{(a} \dot{u}_{b)}-2 n_{(a} \dot{n}_{b)}=2 u_{(a} \mathcal{A}_{b)}-2 n_{(a} \alpha_{b)} \\
\hat{N}_{a b} & =-2 n_{(a} \hat{n}_{b)}, \\
\delta_{c} N_{a b} & =0 \\
\dot{\varepsilon}_{a b} & =-2 u_{[a} \varepsilon_{b] c} \mathcal{A}^{c}+2 n_{[a} \varepsilon_{b] c} \alpha^{c} \\
\hat{\varepsilon}_{a b} & =2 n_{[a} \varepsilon_{b] c} a^{c} \\
\delta_{c} \varepsilon_{a b} & =0 .
\end{aligned}
$$

We now decompose the covariant derivative of $n^{a}$ orthogonal to $u^{a}$ into its irreducible form:

$$
\mathrm{D}_{a} n_{b}=n_{a} a_{b}+\frac{1}{2} \phi N_{a b}+\xi \varepsilon_{a b}+\zeta_{a b}
$$

where

$$
\begin{aligned}
a_{a} & \equiv n^{c} \mathrm{D}_{c} n_{a}=\hat{n}_{a} \\
\phi & \equiv \delta_{a} n^{a} \\
\xi & \equiv \frac{1}{2} \varepsilon^{a b} \delta_{a} n_{b} \\
\zeta_{a b} & \equiv \delta_{\{a} n_{b\}}
\end{aligned}
$$

We may interpret these as follows: travelling along $n^{a}, \phi$ represents the sheet expansion, $\zeta_{a b}$ is the shear of $n^{a}$ (distortion of the sheet), and $a^{a}$ its acceleration, while $\xi$ represents a 'twisting' of the sheet - the rotation of $n^{a}$ [4]. The other derivative of $n^{a}$ is its change along $u^{a}$,

$$
\dot{n}_{a}=\mathcal{A} u_{a}+\alpha_{a} \quad \text { where } \quad \alpha_{a} \equiv \dot{n}_{\bar{a}} \quad \text { and } \quad \mathcal{A}=n^{a} \dot{u}_{a}
$$

The new variables $a_{a}, \phi, \xi, \zeta_{a b}$ and $\alpha_{a}$ are fundamental objects in the spacetime, and their dynamics gives us information about the spacetime geometry. They are treated on the same footing as the kinematical variables of $u^{a}$ in the $1+3$ approach (which also appear here).

For any vector $\Psi^{a}$ orthogonal to $n^{a}$ and $u^{a}$ (i.e., $\Psi^{a}=\Psi^{\bar{a}}$ ), we may decompose the different parts of its spatial derivative:

$$
\mathrm{D}_{a} \Psi_{b}=-n_{a} n_{b} \Psi_{c} a^{c}+n_{a} \hat{\Psi}_{\bar{b}}-n_{b}\left[\frac{1}{2} \phi \Psi_{a}+\left(\xi \varepsilon_{a c}+\zeta_{a c}\right) \Psi^{c}\right]+\delta_{a} \Psi_{b}
$$

Similarly, for a tensor $\Psi_{a b}: \Psi_{a b}=\Psi_{\{a b\}}$, we have

$$
\mathrm{D}_{a} \Psi_{b c}=-2 n_{a} n_{(b} \Psi_{c) d} a^{d}+n_{a} \hat{\Psi}_{b c}-2 n_{(b}\left[\frac{1}{2} \phi \Psi_{c) a}+\Psi_{c)}^{d}\left(\xi \varepsilon_{a d}+\zeta_{a d}\right)\right]+\delta_{a} \Psi_{b c}
$$

Note that for a scalar, we have $\mathrm{D}_{a} \Psi=\hat{\Psi} n_{a}+\delta_{a} \Psi$.

We take $n^{a}$ to be arbitrary at this point, and then split the usual $1+3$ kinematical and Weyl quantities into the irreducible set $\left\{\theta, \mathcal{A}, \Omega, \Sigma, \mathcal{E}, \mathcal{H}, \mathcal{A}^{a}, \Sigma^{a}, \mathcal{E}^{a}, \mathcal{H}^{a}, \Sigma_{a b}, \mathcal{E}_{a b}, \mathcal{H}_{a b}\right\}$ using (3) and (4) as follows:

$$
\begin{aligned}
\dot{u}^{a} & =\mathcal{A} n^{a}+\mathcal{A}^{a} \\
\omega^{a} & =\Omega n^{a}+\Omega^{a} \\
\sigma_{a b} & =\Sigma\left(n_{a} n_{b}-\frac{1}{2} N_{a b}\right)+2 \Sigma_{(a} n_{b)}+\Sigma_{a b}, \\
E_{a b} & =\mathcal{E}\left(n_{a} n_{b}-\frac{1}{2} N_{a b}\right)+2 \mathcal{E}_{(a} n_{b)}+\mathcal{E}_{a b}, \\
H_{a b} & =\mathcal{H}\left(n_{a} n_{b}-\frac{1}{2} N_{a b}\right)+2 \mathcal{H}_{(a} n_{b)}+\mathcal{H}_{a b} .
\end{aligned}
$$


The shear scalar, $\sigma$, for example, may be expressed in the form

$$
\sigma^{2} \equiv \frac{1}{2} \sigma_{a b} \sigma^{a b}=\frac{3}{4} \Sigma^{2}+\Sigma_{a} \Sigma^{a}+\frac{1}{2} \Sigma_{a b} \Sigma^{a b} .
$$

Similarly we may split the fluid variables $q^{a}$ and $\pi_{a b}$,

$$
\begin{aligned}
q^{a} & =Q n^{a}+Q^{a}, \\
\pi_{a b} & =\Pi\left(n_{a} n_{b}-\frac{1}{2} N_{a b}\right)+2 \Pi_{(a} n_{b)}+\Pi_{a b} .
\end{aligned}
$$

Having described the splitting of the $1+3$ variables to obtain their $1+1+2$ parts, and the introduction of the new $1+1+2$ variables corresponding to the irreducible parts of $\nabla_{a} n_{b}$, it only remains to apply this decomposition procedure to the $1+3$ equations themselves, as well as the Ricci identities for $n^{a}$. We give these equations in section III.

\section{A. Commutation relations}

In general the three derivatives we now have defined, '. ', '^' and ' $\delta_{a}$ ' do not commute. Instead, when acting on a scalar $\psi$, they satisfy:

$$
\begin{aligned}
\hat{\dot{\psi}}-\dot{\hat{\psi}} & =-\mathcal{A} \dot{\psi}+\left(\frac{1}{3} \theta+\Sigma\right) \hat{\psi}+\left(\Sigma_{a}+\varepsilon_{a b} \Omega^{b}-\alpha_{a}\right) \delta^{a} \psi, \\
\delta_{a} \dot{\psi}-N_{a}^{b}\left(\delta_{b} \psi\right)^{\cdot} & =-\mathcal{A}_{a} \dot{\psi}+\left(\alpha_{a}+\Sigma_{a}-\varepsilon_{a b} \Omega^{b}\right) \hat{\psi}+\left(\frac{1}{3} \theta-\frac{1}{2} \Sigma\right) \delta_{a} \psi+\left(\Sigma_{a b}+\Omega \varepsilon_{a b}\right) \delta^{b} \psi, \\
\delta_{a} \hat{\psi}-N_{a}{ }^{b}\left(\widehat{\delta_{b} \psi}\right) & =\left(\Sigma_{a}-\varepsilon_{a b} \Omega^{b}\right) \dot{\psi}+a_{a} \hat{\psi}+\frac{1}{2} \phi \delta_{a} \psi+\left(\zeta_{a b}+\xi \varepsilon_{a b}\right) \delta^{b} \psi \\
\delta_{a} \delta_{b} \psi-\delta_{b} \delta_{a} \psi & =2 \varepsilon_{a b}(\Omega \dot{\psi}-\xi \hat{\psi})+2 a_{[a} \delta_{b]} \psi .
\end{aligned}
$$

The commutation relations for 2 -vectors $\psi_{a}$ are

$$
\begin{aligned}
\hat{\dot{\psi}}_{\bar{a}}-\dot{\hat{\psi}}_{\bar{a}}= & -\mathcal{A} \dot{\psi}_{\bar{a}}+\left(\frac{1}{3} \theta+\Sigma\right) \hat{\psi}_{\bar{a}}+\left(\Sigma_{b}+\varepsilon_{b c} \Omega^{c}-\alpha_{b}\right) \delta^{b} \psi_{a}+\mathcal{A}_{a}\left(\Sigma_{b}+\varepsilon_{b c} \Omega^{c}\right) \psi^{b}+\mathcal{H}_{a b} \psi^{b}, \\
\delta_{a} \dot{\psi}_{b}-N_{a}{ }^{c} N_{b}{ }^{d}\left(\delta_{c} \psi_{d}\right)^{\cdot}= & -\mathcal{A}_{a} \dot{\psi}_{b}+\left(\alpha_{a}+\Sigma_{a}-\varepsilon_{a c} \Omega^{c}\right) \hat{\psi}_{\bar{b}}+\left(\frac{1}{3} \theta-\frac{1}{2} \Sigma\right)\left(\delta_{a} \psi_{b}+\psi_{a} \mathcal{A}_{b}\right)+\mathcal{H}_{a} \varepsilon_{b c} \psi^{c} \\
& +\left(\Sigma_{a c}+\Omega \varepsilon_{a c}\right)\left(\delta^{c} \psi_{b}+\psi^{c} \mathcal{A}_{b}\right)+\frac{1}{2}\left(\psi_{a} Q_{b}-N_{a b} \psi^{c} Q_{c}\right) \\
& -\left(\frac{1}{2} \phi N_{a c}+\xi \varepsilon_{a c}+\zeta_{a c}\right) \psi^{c} \alpha_{b} \\
\delta_{a} \hat{\psi}_{b}-N_{a}{ }^{c} N_{b}{ }^{d}\left(\widehat{\delta}_{c} \psi_{d}\right)= & \left(\Sigma_{a}-\varepsilon_{a c} \Omega^{c}\right) \dot{\psi}_{\bar{b}}+a_{a} \hat{\psi}_{\bar{b}}+\frac{1}{2} \phi\left(\delta_{a} \psi_{b}-\psi_{a} a_{b}\right)+\left(\zeta_{a c}+\xi \varepsilon_{a c}\right)\left(\delta^{c} \psi_{b}-\psi^{c} a_{b}\right) \\
& +N_{a b} \psi^{c}\left(\frac{1}{2} \Pi_{c}+\mathcal{E}_{c}\right)-\psi_{a}\left(\frac{1}{2} \Pi_{b}+\mathcal{E}_{b}\right) \\
\delta_{a} \delta_{b} \psi_{c}-\delta_{b} \delta_{a} \psi_{c}= & 2 \varepsilon_{a b}\left(\Omega \dot{\psi}_{\bar{c}}-\xi \hat{\psi}_{\bar{c}}\right)+2\left[\left(\frac{1}{3} \theta-\frac{1}{2} \Sigma\right)^{2}-\frac{1}{4} \phi^{2}+\frac{1}{2} \Pi+\mathcal{E}-\frac{1}{3}(\mu+\Lambda)\right] \psi_{[a} N_{b] c} \\
& -2 \psi_{[a}\left[-\left(\frac{1}{3} \theta-\frac{1}{2} \Sigma\right)\left(\Sigma_{b] c}+\Omega \varepsilon_{b] c}\right)+\frac{1}{2} \phi\left(\zeta_{b] c}+\xi \varepsilon_{b] c}\right)+\frac{1}{2} \Pi_{b] c}+\mathcal{E}_{b] c}\right] \\
& +2 N_{[a c}\left[-\left(\frac{1}{3} \theta-\frac{1}{2} \Sigma\right)\left(\Sigma_{b] d}+\Omega \varepsilon_{b] d}\right)+\frac{1}{2} \phi\left(\zeta_{b] d}+\xi \varepsilon_{b] d}\right)+\frac{1}{2} \Pi_{b] d}+\mathcal{E}_{b] d}\right] \psi^{d} \\
& +2\left[-\left(\Sigma_{[a c}+\Omega \varepsilon_{[a c}\right)\left(\Sigma_{b] d}+\Omega \varepsilon_{b] d}\right)+\left(\zeta_{[a c}+\xi \varepsilon_{[a c}\right)\left(\zeta_{b] d}+\xi \varepsilon_{b] d}\right)\right] \psi^{d}
\end{aligned}
$$

These relations are more complicated for tensors. These last two equations in the case of scalars are the decomposition of the $1+3$ commutation relation

$$
\operatorname{curl} \mathrm{D}_{a} \psi=2 \dot{\psi} \omega_{a}
$$

From Eq. (37), we see that our sheet will be a genuine 2-surface in the spacetime (and, in particular, that the derivative $\delta_{a}$ will be a true covariant derivative on this surface) if and only if $\xi=\Omega=a^{a}=0$. (Recall that the $1+3$ spatial metric $h_{a b}$ corresponds to a genuine 3 -surface when $\omega^{a}=0$.) Otherwise, the sheet is really just a collection of tangent planes. In addition, the two vectors $u^{a}$ and $n^{a}$ are 2 -surface forming if and only if the commutator $[u, n]$ in (34) has no component in the sheet: that is, when Greenberg's vector

$$
\Sigma^{a}+\varepsilon^{a b} \Omega_{b}-\alpha^{a}
$$

vanishes [16] - see Eq. (34). 


\section{THE EQUATIONS}

Once the vector $n^{a}$ has been introduced it is possible, and necessary, to augment the $1+3$ equations with the Ricci identities for $n^{a}$; without these we do not have enough equations to determine the new $1+1+2$ variables. The Ricci identities for $n^{a}$ are

$$
R_{a b c} \equiv 2 \nabla_{[a} \nabla_{b]} n_{c}-R_{a b c d} n^{d}=0,
$$

where $R_{a b c d}$ is the Riemann curvature tensor. This third-rank tensor may be covariantly split using the two vector fields $u^{a}$ and $n^{a}$, and gives dynamical equations for the covariant parts of the derivative of $n^{a}$ (namely $\alpha_{a}, a_{a}, \phi, \xi$ and $\left.\zeta_{a b}\right)$ in the form of evolution equations, involving dot-derivatives of these variables, and propagation equations, involving hat-derivatives. In order to facilitate the calculation of these Ricci identities, which appear in the following section, we give here the expression for the full covariant derivative of $n^{a}$ in terms of the relevant $1+1+2$ variables:

$$
\nabla_{a} n_{b}=-\mathcal{A} u_{a} u_{b}-u_{a} \alpha_{b}+\left(\Sigma+\frac{1}{3} \theta\right) n_{a} u_{b}+\left(\Sigma_{a}-\varepsilon_{a c} \Omega^{c}\right) u_{b}+n_{a} a_{b}+\frac{1}{2} \phi N_{a b}+\xi \varepsilon_{a b}+\zeta_{a b}
$$

which may be inserted into Eq. (44). The full decomposition of the covariant derivative of $u^{a}$ is

$$
\begin{aligned}
\nabla_{a} u_{b}= & -u_{a}\left(\mathcal{A} n_{b}+\mathcal{A}_{b}\right)+n_{a} n_{b}\left(\frac{1}{3} \theta+\Sigma\right)+n_{a}\left(\Sigma_{b}+\varepsilon_{b c} \Omega^{c}\right) \\
& +\left(\Sigma_{a}-\varepsilon_{a c} \Omega^{c}\right) n_{b}+N_{a b}\left(\frac{1}{3} \theta-\frac{1}{2} \Sigma\right)+\Omega \varepsilon_{a b}+\Sigma_{a b},
\end{aligned}
$$

which in turn implies the useful relation

$$
\hat{u}_{a}=\left(\frac{1}{3} \theta+\Sigma\right) n_{a}+\Sigma_{a}+\varepsilon_{a b} \Omega^{b} .
$$

We have now assembled all the tools necessary to provide the full system of equations for the $1+1+2$ formalism. This consists of evolution equations, propagation equations, mixtures of both, and constraints. Formulae which are useful for splitting $1+3$ equations are given in Appendix A.

\section{A. Evolution equations}

We find evolution equations for the $1+1+2$ variables $\phi, \xi$ and $\zeta_{a b}$ from the projection $u^{a} R_{a b c}$.

$u^{a} N^{b c} R_{a b c}$ :

$$
\dot{\phi}=\left(\frac{2}{3} \theta-\Sigma\right)\left(\mathcal{A}-\frac{1}{2} \phi\right)+2 \xi \Omega+\delta_{a} \alpha^{a}+\mathcal{A}^{a}\left(\alpha_{a}-a_{a}\right)+\left(a^{a}-\mathcal{A}^{a}\right)\left(\Sigma_{a}-\varepsilon_{a b} \Omega^{b}\right)-\zeta^{a b} \Sigma_{a b}+Q,
$$

$u^{a} \varepsilon^{b c} R_{a b c}:$

$$
\dot{\xi}=\left(\frac{1}{2} \Sigma-\frac{1}{3} \theta\right) \xi+\left(\mathcal{A}-\frac{1}{2} \phi\right) \Omega+\frac{1}{2}\left(a^{a}+\mathcal{A}^{a}\right)\left[\Omega_{a}+\varepsilon_{a b}\left(\alpha^{b}+\Sigma^{b}\right)\right]+\frac{1}{2} \varepsilon_{a b} \delta^{a} \alpha^{b}-\frac{1}{2} \varepsilon_{c a} \zeta_{b}{ }^{c} \Sigma^{a b}+\frac{1}{2} \mathcal{H},
$$

$u^{c} R_{c\{a b\}}:$

$$
\begin{aligned}
\dot{\zeta}_{\{a b\}}= & \left(\frac{1}{2} \Sigma-\frac{1}{3} \theta\right) \zeta_{a b}+\Omega \varepsilon_{c\{a} \zeta_{b\}}{ }^{c}+\left(\mathcal{A}-\frac{1}{2} \phi\right) \Sigma_{a b}-\xi \varepsilon_{c\{a} \Sigma_{b\}}{ }^{c}-\zeta_{c\{a} \Sigma_{b\}}{ }^{c}+\delta_{\{a} \alpha_{b\}} \\
& +\left(\mathcal{A}_{\{a}-a_{\{a}\right) \alpha_{b\}}-\left(\mathcal{A}_{\{a}+a_{\{a}\right)\left(\Sigma_{b\}}-\varepsilon_{b\} d} \Omega^{d}\right)-\varepsilon_{c\{a} \mathcal{H}_{b\}}{ }^{c},
\end{aligned}
$$

Then a $1+1+2$ decomposition of the standard $1+3$ evolution equations gives us the remaining evolution equations, which can't be found from $R_{a b c}$.

Vorticity evolution equation:

$$
\dot{\Omega}=\frac{1}{2} \varepsilon_{a b} \delta^{a} \mathcal{A}^{b}+\mathcal{A} \xi+\Omega\left(\Sigma-\frac{2}{3} \theta\right)+\Omega_{a}\left(\Sigma^{a}+\alpha^{a}\right)
$$

Shear evolution:

$$
\dot{\Sigma}_{\{a b\}}=\delta_{\{a} \mathcal{A}_{b\}}+\mathcal{A}_{\{a} \mathcal{A}_{b\}}-\Sigma_{\{a}\left[\Sigma_{b\}}+2 \alpha_{b\}}\right]-\Omega_{\{a} \Omega_{b\}}+\mathcal{A} \zeta_{a b}-\left(\frac{2}{3} \theta+\frac{1}{2} \Sigma\right) \Sigma_{a b}-\Sigma_{c\{a} \Sigma_{b\}}{ }^{c}-\mathcal{E}_{a b}+\frac{1}{2} \Pi_{a b}
$$




\section{B. Mixture of propagation and evolution:}

$u^{a} n^{b} R_{a b \bar{c}}=n^{a} u^{b} R_{a b \bar{c}}:$

$$
\begin{aligned}
\hat{\alpha}_{\bar{a}}-\dot{a}_{\bar{a}}= & -\left(\frac{1}{2} \phi+\mathcal{A}\right) \alpha_{a}-\xi \varepsilon_{a b} \alpha^{b}+\left(\frac{1}{3} \theta+\Sigma\right)\left(\mathcal{A}_{a}-a_{a}\right)+\left(\frac{1}{2} \phi-\mathcal{A}\right)\left(\Sigma_{a}+\varepsilon_{a b} \Omega^{b}\right) \\
& -\xi\left(\varepsilon_{a b} \Sigma^{b}-\Omega_{a}\right)+\zeta_{a b}\left(-\alpha^{b}+\Sigma^{b}+\varepsilon^{b c} \Omega_{c}\right)+\frac{1}{2} Q_{a}-\varepsilon_{a b} \mathcal{H}^{b},
\end{aligned}
$$

$u^{a} n^{b} u^{c} R_{a b c}=-n^{a} u^{b} u^{c} R_{a b c}$ :

$$
\begin{aligned}
\hat{\mathcal{A}}-\frac{1}{3} \dot{\theta}-\dot{\Sigma}= & -\mathcal{A}^{2}+\left(\frac{1}{3} \theta+\Sigma\right)^{2}-2 \alpha_{a} \Sigma^{a}+\Sigma_{a} \Sigma^{a}-\Omega_{a} \Omega^{a}-a_{a} \mathcal{A}^{a}+\varepsilon_{a b} \alpha^{a} \Omega^{b} \\
& +\frac{1}{6}(\mu+3 p-2 \Lambda)+\mathcal{E}-\frac{1}{2} \Pi
\end{aligned}
$$

Raychaudhuri equation:

$$
\begin{aligned}
\hat{\mathcal{A}}-\dot{\theta}= & -\delta_{a} \mathcal{A}^{a}-(\mathcal{A}+\phi) \mathcal{A}+\left(a_{a}-\mathcal{A}_{a}\right) \mathcal{A}^{a}+\frac{1}{3} \theta^{2}+\frac{3}{2} \Sigma^{2}-2 \Omega^{2}+2 \Sigma_{a} \Sigma^{a} \\
& -2 \Omega_{a} \Omega^{a}+\Sigma_{a b} \Sigma^{a b}+\frac{1}{2}(\mu+3 p)-\Lambda
\end{aligned}
$$

Vorticity evolution:

$$
\begin{aligned}
\dot{\Omega}_{\bar{a}}+\frac{1}{2} \varepsilon_{a b} \hat{\mathcal{A}}^{b}= & -\left(\frac{2}{3} \theta+\frac{1}{2} \Sigma\right) \Omega_{a}+\Omega\left(\Sigma_{a}-\alpha_{a}\right)+\frac{1}{2} \xi \mathcal{A}_{a}+\frac{1}{2} \varepsilon_{a b}\left[-\mathcal{A} a^{b}+\delta^{b} \mathcal{A}-\frac{1}{2} \phi \mathcal{A}^{b}\right] \\
& -\frac{1}{2} \varepsilon_{a b} \zeta^{b c} \mathcal{A}_{c}+\Sigma_{a b} \Omega^{b}
\end{aligned}
$$

Shear evolution:

$$
\begin{aligned}
\dot{\Sigma}-\frac{2}{3} \hat{\mathcal{A}}= & \frac{1}{3}(2 \mathcal{A}-\phi) \mathcal{A}-\left(\frac{2}{3} \theta+\frac{1}{2} \Sigma\right) \Sigma-\frac{2}{3} \Omega^{2}-\frac{1}{3} \delta_{a} \mathcal{A}^{a}+\Sigma_{a}\left[2 \alpha^{a}-\frac{1}{3} \Sigma^{a}\right] \\
& -\frac{1}{3} \mathcal{A}_{a}\left[2 a^{a}-\mathcal{A}^{a}\right]+\frac{1}{3} \Omega_{a} \Omega^{a}+\frac{1}{3} \Sigma_{a b} \Sigma^{a b}-\mathcal{E}+\frac{1}{2} \Pi \\
\dot{\Sigma}_{\bar{a}}-\frac{1}{2} \hat{\mathcal{A}}_{\bar{a}}= & \frac{1}{2} \delta_{a} \mathcal{A}+\left(\mathcal{A}-\frac{1}{4} \phi\right) \mathcal{A}_{a}-\left(\frac{2}{3} \theta+\frac{1}{2} \Sigma\right) \Sigma_{a}+\frac{1}{2} \mathcal{A} a_{a}-\frac{3}{2} \Sigma \alpha_{a}-\Omega \Omega_{a} \\
& -\frac{1}{2}\left(\xi \varepsilon_{a b}+\zeta_{a b}\right) \mathcal{A}^{b}+\Sigma_{a b}\left(\alpha^{b}-\Sigma^{b}\right)-\mathcal{E}_{a}+\frac{1}{2} \Pi_{a}
\end{aligned}
$$

Energy conservation:

$$
\dot{\mu}+\hat{Q}=-\delta_{a} Q^{a}-\theta(\mu+p)-(\phi+2 \mathcal{A}) Q-\frac{3}{2} \Sigma \Pi+\left(a_{a}-2 \mathcal{A}_{a}\right) Q^{a}-2 \Sigma_{a} \Pi^{a}-\Sigma_{a b} \Pi^{a b}
$$

Momentum conservation:

$$
\begin{gathered}
\dot{Q}+\hat{p}+\hat{\Pi}=-\delta_{a} \Pi^{a}-\left(\frac{3}{2} \phi+\mathcal{A}\right) \Pi-\left(\frac{4}{3} \theta+\Sigma\right) Q-(\mu+p) \mathcal{A} \\
+\left(\alpha_{a}-\Sigma_{a}+\varepsilon_{a b} \Omega^{b}\right) Q^{a}+\left(2 a_{a}-\mathcal{A}_{a}\right) \Pi^{a}+\zeta_{a b} \Pi^{a b} \\
\dot{Q}_{\bar{a}}+\hat{\Pi}_{\bar{a}}=-\delta_{a} p+\frac{1}{2} \delta_{a} \Pi-\delta^{b} \Pi_{a b}-Q\left(\alpha_{a}+\Sigma_{a}+\varepsilon_{a b} \Omega^{b}\right)-\frac{3}{2} \Pi a_{a}-\left(\frac{4}{3} \theta-\frac{1}{2} \Sigma\right) Q_{a}+\Omega \varepsilon_{a b} Q^{b} \\
-\left(\frac{3}{2} \phi+\mathcal{A}\right) \Pi_{a}+\xi \varepsilon_{a b} \Pi^{b}-\left(\mu+p-\frac{1}{2} \Pi\right) \mathcal{A}_{a}-\Sigma_{a b} Q^{b}-\zeta_{a b} \Pi^{b}+\Pi_{a b}\left(a^{b}-\mathcal{A}^{b}\right)
\end{gathered}
$$

Electric Weyl evolution:

$$
\begin{aligned}
\dot{\mathcal{E}}+\frac{1}{2} \dot{\Pi}+\frac{1}{3} \hat{Q}= & +\varepsilon_{a b} \delta^{a} \mathcal{H}^{c}+\frac{1}{6} \delta_{a} Q^{a}+\left(\frac{3}{2} \Sigma-\theta\right) \mathcal{E}-\frac{1}{2}\left(\frac{1}{3} \theta+\frac{1}{2} \Sigma\right) \Pi+\frac{1}{3}\left(\frac{1}{2} \phi-2 \mathcal{A}\right) Q+3 \xi \mathcal{H} \\
& -\frac{1}{2}(\mu+p) \Sigma+\left(2 \alpha_{a}+\Sigma_{a}-\varepsilon_{a b} \Omega^{b}\right) \mathcal{E}^{a}+\left(\alpha_{a}-\frac{1}{6} \Sigma_{a}-\frac{1}{2} \varepsilon_{a b} \Omega^{b}\right) \Pi^{a} \\
& +\frac{1}{3}\left(a_{a}+\mathcal{A}_{a}\right) Q^{a}+2 \varepsilon_{a b} \mathcal{A}^{a} \mathcal{H}^{c}-\Sigma_{a b}\left(\mathcal{E}^{a b}+\frac{1}{2} \Pi^{a b}\right)+\varepsilon_{a b} \mathcal{H}^{b c} \zeta_{c}^{a} \\
\dot{\mathcal{E}}_{\bar{a}}+\frac{1}{2} \varepsilon_{a b} \hat{\mathcal{H}}^{b}+\frac{1}{2} \dot{\Pi}_{\bar{a}}+\frac{1}{4} \hat{Q}_{\bar{a}}= & \frac{3}{4} \varepsilon_{a b} \delta^{b} \mathcal{H}+\frac{1}{2} \varepsilon_{b c} \delta^{b} \mathcal{H}^{c}{ }_{a}-\frac{1}{4} \delta_{a} Q-\frac{1}{2}\left(\mu+p-\frac{3}{2} \mathcal{E}+\frac{1}{4} \Pi\right) \Sigma_{a} \\
& +\frac{3}{4}\left(\mathcal{E}+\frac{1}{2} \Pi\right) \varepsilon_{a b} \Omega^{b}-\frac{1}{2} Q \mathcal{A}_{a}+\frac{3}{2} \mathcal{H} \varepsilon_{a b} \mathcal{A}^{b}-\frac{3}{2}\left(\mathcal{E}+\frac{1}{2} \Pi\right) \alpha_{a} \\
& -\frac{1}{4} Q a_{a}-\frac{3}{4} \mathcal{H} \varepsilon_{a b} a^{b}+\left(\frac{3}{4} \Sigma-\theta\right) \mathcal{E}_{a}-\frac{1}{2} \Omega \varepsilon_{a b} \mathcal{E}^{b}+\frac{5}{2} \xi \mathcal{H}_{a}-\left(\frac{1}{4} \phi+\mathcal{A}\right) \varepsilon_{a b} \mathcal{H}^{b} \\
& +\frac{1}{2}\left(\frac{1}{4} \phi-\mathcal{A}\right) Q_{a}+\frac{1}{4} \xi \varepsilon_{a b} Q^{b}-\frac{1}{2}\left(\frac{1}{3} \theta+\frac{1}{4} \Sigma\right) \Pi_{a}-\frac{1}{4} \Omega \varepsilon_{a b} \Pi^{b} \\
& +\frac{1}{2} \Sigma_{a b}\left(3 \mathcal{E}^{b}-\frac{1}{2} \Pi^{b}\right)+\frac{1}{2}\left(3 \mathcal{E}_{a b}-\frac{1}{2} \Pi_{a b}\right) \Sigma^{b}-\left(\mathcal{E}_{a b}+\frac{1}{2} \Pi_{a b}\right)\left(\alpha^{b}+\frac{1}{2} \varepsilon^{b c} \Omega_{c}\right) \\
& +\frac{1}{2} \zeta_{a b}\left(\varepsilon^{b c} \mathcal{H}_{c}+Q^{b}\right)-\mathcal{H}_{a b} \varepsilon^{b c} \mathcal{A}_{c}
\end{aligned}
$$




$$
\begin{aligned}
\dot{\mathcal{E}}_{\{a b\}}-\varepsilon_{c\{a} \hat{\mathcal{H}}_{b\}}^{c}+\frac{1}{2} \dot{\Pi}_{\{a b\}}= & -\varepsilon_{c\{a} \delta^{c} \mathcal{H}_{b\}}-\frac{1}{2} \delta_{\{a} Q_{b\}}-\frac{1}{2}\left(\mu+p+3 \mathcal{E}-\frac{1}{2} \Pi\right) \Sigma_{a b}-\frac{1}{2} Q \zeta_{a b}-\frac{3}{2} \mathcal{H} \varepsilon_{c\{a} \zeta_{b\}}{ }^{c} \\
& -\left(\theta+\frac{3}{2} \Sigma\right) \mathcal{E}_{a b}+\Omega \varepsilon_{c\{a} \mathcal{E}_{b\}}{ }^{c}-\left(\frac{1}{6} \theta-\frac{1}{4} \Sigma\right) \Pi_{a b}+\frac{1}{2} \Omega \varepsilon_{c\{a} \Pi_{b\}}{ }^{c}+\xi \mathcal{H}_{a b} \\
& +\left(\frac{1}{2} \phi+2 \mathcal{A}\right) \varepsilon_{c\{a} \mathcal{H}_{b\}}{ }^{c}-\mathcal{A}_{\{a} Q_{b\}}-\left(\alpha_{\{a}+\frac{1}{2} \varepsilon_{c\{a} \Omega^{c}\right)\left(2 \mathcal{E}_{b\}}+\Pi_{b\}}\right) \\
& +\Sigma_{\{a}\left(3 \mathcal{E}_{b\}}-\frac{1}{2} \Pi_{b\}}\right)+2 \varepsilon_{c\{a} \mathcal{H}_{b\}}\left(a^{c}-\mathcal{A}^{c}\right) \\
& +\Sigma_{c\{a}\left(3 \mathcal{E}_{b\}}^{c}-\frac{1}{2} \Pi_{b\}}{ }^{c}\right)+\varepsilon_{c\{a} \mathcal{H}_{b\} d} \zeta^{c d}
\end{aligned}
$$

Magnetic Weyl evolution:

$$
\begin{aligned}
& \dot{\mathcal{H}}=--\varepsilon_{a b} \delta^{a} \mathcal{E}^{b}+\frac{1}{2} \varepsilon_{a b} \delta^{a} \Pi^{b}-3 \xi \mathcal{E}+\left(\theta+\frac{3}{2} \Sigma\right) \mathcal{H}+\Omega Q+\frac{3}{2} \xi \Pi-2 \varepsilon_{a b} \mathcal{A}^{a} \mathcal{E}^{b} \\
&+\left(2 \alpha_{a}+\Sigma_{a}-\varepsilon_{a b} \Omega^{b}\right) \mathcal{H}^{a}-\frac{1}{2}\left(\Omega_{a}+\varepsilon_{a b} \Sigma^{b}\right) Q^{a}-\Sigma_{a b} \mathcal{H}^{a b}-\frac{1}{2} \varepsilon_{a b} \mathcal{E}^{b c} \zeta_{c}^{a} \\
& \dot{\mathcal{H}}_{\bar{a}}-\frac{1}{2} \varepsilon_{a b} \hat{\mathcal{E}}^{b}+\frac{1}{4} \varepsilon_{a b} \hat{\Pi}^{b}=-\frac{3}{4} \varepsilon_{a b} \delta^{b} \mathcal{E}+\frac{3}{8} \varepsilon_{a b} \delta^{b} \Pi-\frac{1}{2} \varepsilon_{b c} \delta^{b} \mathcal{E}_{a}^{c}+\frac{1}{4} \varepsilon_{b c} \delta^{b} \Pi^{c} \\
&+ \frac{3}{4} \mathcal{H} \Sigma_{a}+\frac{1}{4} Q \varepsilon_{a b} \Sigma^{b}+\frac{3}{4} Q \Omega_{a}+\frac{3}{4} \mathcal{H} \varepsilon_{a b} \Omega^{b}-\frac{3}{2} \mathcal{E}_{a b} \mathcal{A}^{b}-\frac{3}{2} \mathcal{H} \alpha_{a} \\
&+ \frac{3}{4}\left(\mathcal{E}-\frac{1}{2} \Pi\right) \varepsilon_{a b} a^{b}-\frac{5}{2} \xi \mathcal{E}_{a}+\left(\frac{1}{4} \phi+\mathcal{A}\right) \varepsilon_{a b} \mathcal{E}^{b}+\left(\frac{3}{4} \Sigma-\theta\right) \mathcal{H}_{a}-\frac{1}{2} \Omega \varepsilon_{a b} \mathcal{H}^{b} \\
&+\frac{3}{4} \Omega Q_{a}-\frac{3}{8} \Sigma \varepsilon_{a b} Q^{b}+\frac{5}{4} \xi \Pi_{a}-\frac{1}{8} \phi \varepsilon_{a b} \Pi^{b}+\Sigma_{a b}\left(\frac{3}{2} \mathcal{H}^{b}+\frac{1}{4} \varepsilon^{b c} Q_{c}\right) \\
&+\frac{3}{2} \varepsilon_{a b} \zeta^{b c}\left(\mathcal{E}_{c}-\frac{1}{2} \Pi_{c}+\frac{2}{3} \mathcal{A}_{c}\right)+\mathcal{H}_{a b}\left(\alpha^{b}+\frac{3}{2} \Sigma^{b}-\frac{1}{2} \varepsilon^{b c} \Omega_{c}\right) \\
& \dot{\mathcal{H}}_{\{a b\}}+\varepsilon_{c\{a} \hat{\mathcal{E}}_{b\}}{ }^{c}-\frac{1}{2} \varepsilon_{c\{a} \hat{\Pi}_{b\}}{ }^{c}= \varepsilon_{c\{a} \delta^{c} \mathcal{E}_{b\}}-\frac{1}{2} \varepsilon_{c\{a} \delta^{c} \Pi_{b\}}-\frac{3}{2} \mathcal{H} \Sigma_{a b}+\frac{1}{2} Q \varepsilon_{c\{a} \Sigma_{b\}}{ }^{c} \\
&+\frac{3}{2}\left(\mathcal{E}-\frac{1}{2} \Pi\right) \varepsilon_{c\{a} \zeta_{b\}}{ }^{c}-\xi \mathcal{E}_{a b}-\left(\frac{1}{2} \phi+2 \mathcal{A}\right) \varepsilon_{c\{a} \mathcal{E}_{b\}}{ }^{c} \\
&-\left(\theta+\frac{3}{2} \Sigma\right) \mathcal{H}_{a b}-\Omega \varepsilon_{c\{a} \mathcal{H}_{b\}}{ }^{c}+\frac{1}{2} \xi \Pi_{a b}+\frac{1}{4} \phi \varepsilon_{c\{a} \Pi_{b\}}{ }^{c} \\
&+\Sigma_{\{a}\left(3 \mathcal{H}_{b\}}-\varepsilon_{b\} c} Q^{c}\right)+\Omega_{\{a}\left(\frac{3}{2} Q_{b\}}-\varepsilon_{b\} c} H^{c}\right)-2 \alpha_{\{a} \mathcal{H}_{b\}} \\
&+\mathcal{E}_{\{a} 2 \varepsilon_{b\} c}\left(a^{c}+\mathcal{A}^{c}\right)-\Pi_{\{a} \varepsilon_{b\} c} a^{c}+3 \Sigma_{c\{a} \mathcal{H}_{b\}}{ }^{c}-\varepsilon_{c\{a} \zeta^{c d}\left(\mathcal{E}_{b\} d}-\frac{1}{2} \Pi_{b\} d}\right)
\end{aligned}
$$

\section{Propagation equations}

Propagation and constraint equations are formed from either projecting $R_{a b c}$ as indicated, or from projections of the $1+3$ constraint equations, denoted $C_{i}$, as given in [1].

$n^{a} N^{b c} R_{a b c}$ :

$$
\begin{aligned}
\hat{\phi}= & -\frac{1}{2} \phi^{2}+2 \xi^{2}+\left(\frac{1}{3} \theta+\Sigma\right)\left(\frac{2}{3} \theta-\Sigma\right)+\delta_{a} a^{a}-a_{a} a^{a}-\zeta_{a b} \zeta^{a b}+2 \varepsilon_{a b} \alpha^{a} \Omega^{b}-\Sigma_{a} \Sigma^{a}+\Omega_{a} \Omega^{a} \\
& -\frac{2}{3}(\mu+\Lambda)-\frac{1}{2} \Pi-\mathcal{E},
\end{aligned}
$$

$n^{a} \varepsilon^{b c} R_{a b c}$

$$
\hat{\xi}=-\phi \xi+\left(\frac{1}{3} \theta+\Sigma\right) \Omega+\frac{1}{2} \varepsilon_{a b} \delta^{a} a^{b}+\frac{1}{2} \varepsilon_{a b} \Sigma^{a} a^{b}+\left(\frac{1}{2} a_{a}+\alpha_{a}\right) \Omega^{a}
$$

$n^{a} R_{a\{b c\}}$

$$
\begin{aligned}
\hat{\zeta}_{\{a b\}}= & -\phi \zeta_{a b}-\zeta_{\{a}^{c} \zeta_{b\} c}+\delta_{\{a} a_{b\}}-a_{\{a} a_{b\}}+2 \alpha_{\{a} \varepsilon_{b\} c} \Omega^{c}-\Omega_{\{a} \Omega_{b\}}-\Sigma_{\{a} \Sigma_{b\}} \\
& +\left(\frac{1}{3} \theta+\Sigma\right) \Sigma_{a b}-\frac{1}{2} \Pi_{a b}-\mathcal{E}_{a b},
\end{aligned}
$$

Shear divergence $\left(C_{1}\right)^{a} n_{a}$ :

$$
\hat{\Sigma}-\frac{2}{3} \hat{\theta}=-\frac{3}{2} \phi \Sigma-2 \xi \Omega-\delta_{a} \Sigma^{a}-\varepsilon_{a b} \delta^{a} \Omega^{b}+2 \Sigma_{a} a^{a}-2 \varepsilon_{a b} \mathcal{A}^{a} \Omega^{b}+\Sigma_{a b} \zeta^{a b}-Q
$$


and $\left(C_{1}\right)_{\bar{a}}$ :

$$
\begin{aligned}
\hat{\Sigma}_{\bar{a}}-\varepsilon_{a b} \hat{\Omega}^{b}= & \frac{1}{2} \delta_{a} \Sigma+\frac{2}{3} \delta_{a} \theta-\varepsilon_{a b} \delta^{b} \Omega-\frac{3}{2} \phi \Sigma_{a}+\xi \varepsilon_{a b} \Sigma^{b}-\xi \Omega_{a}+\left(\frac{1}{2} \phi+2 \mathcal{A}\right) \varepsilon_{a b} \Omega^{b} \\
& -\frac{3}{2} \Sigma a_{a}+\Omega \varepsilon_{a b}\left[a^{b}-2 \mathcal{A}^{b}\right]-\delta^{b} \Sigma_{a b}-\zeta_{a b} \Sigma^{b}+\Sigma_{a b} a^{b}+\varepsilon_{a b} \zeta^{b c} \Omega_{c}-Q_{a}
\end{aligned}
$$

Vorticity divergence equation $\left(C_{2}\right)$ :

$$
\hat{\Omega}=-\delta_{a} \Omega^{a}+(\mathcal{A}-\phi) \Omega+\left(a_{a}+\mathcal{A}_{a}\right) \Omega^{a}
$$

$\left(C_{3}\right)_{\{a b\}}:$

$$
\begin{aligned}
\hat{\Sigma}_{\{a b\}}= & \delta_{\{a} \Sigma_{b\}}-\varepsilon_{c\{a} \delta^{c} \Omega_{b\}}-\frac{1}{2} \phi \Sigma_{a b}+\xi \varepsilon_{c\{a} \Sigma_{b\}}{ }^{c}+\frac{3}{2} \Sigma \zeta_{a b}-\Omega \varepsilon_{c\{a} \zeta_{b\}}{ }^{c} \\
& -2 \Sigma_{\{a} a_{b\}}-2 \varepsilon_{c\{a} \mathcal{A}^{c} \Omega_{b\}}-\Sigma_{c\{a} \zeta_{b\}}{ }^{c}-\varepsilon_{c\{a} \mathcal{H}_{b\}}{ }^{c}
\end{aligned}
$$

Electric Weyl Divergence $\left(C_{4}\right)^{a} n_{a}$ :

$$
\begin{aligned}
\hat{\mathcal{E}}-\frac{1}{3} \hat{\mu}+\frac{1}{2} \hat{\Pi}= & -\delta_{a} \mathcal{E}^{a}-\frac{1}{2} \delta_{a} \Pi^{a}-\frac{3}{2} \phi\left(\mathcal{E}+\frac{1}{2} \Pi\right)+\left(\frac{1}{2} \Sigma-\frac{1}{3} \theta\right) Q+3 \Omega \mathcal{H}+\left(2 \mathcal{E}_{a}+\Pi_{a}\right) a^{a} \\
& +\frac{1}{2} \Sigma_{a} Q^{a}+3 \Omega_{a} \mathcal{H}^{a}-\frac{3}{2} \varepsilon_{a b} \Omega^{a} Q^{b}+\varepsilon_{a b} \Sigma^{a c} \mathcal{H}_{c}^{b}+\left(\mathcal{E}_{a b}+\frac{1}{2} \Pi_{a b}\right) \zeta^{a b}
\end{aligned}
$$

$\left(C_{4}\right)_{\bar{a}}:$

$$
\begin{aligned}
\hat{\mathcal{E}}_{\bar{a}}+\frac{1}{2} \hat{\Pi}_{\bar{a}}= & \frac{1}{2} \delta_{a} \mathcal{E}+\frac{1}{3} \delta_{a} \mu+\frac{1}{4} \delta_{a} \Pi-\delta^{b} \mathcal{E}_{a b}-\frac{1}{2} \delta^{b} \Pi_{a b}+\frac{1}{2} Q \Sigma_{a}+\mathcal{H} \varepsilon_{a b} \Sigma^{b}-\frac{3}{2} \mathcal{H} \Omega_{a}-\frac{3}{2} Q \varepsilon_{a b} \Omega^{b} \\
& -\frac{3}{2}\left(\mathcal{E}+\frac{1}{2} \Pi\right) a_{a}-\frac{3}{2} \phi\left(\mathcal{E}_{a}+\frac{1}{2} \Pi_{a}\right)+\xi \varepsilon_{a b}\left(\mathcal{E}^{b}+\frac{1}{2} \Pi^{b}\right)+3 \Omega \mathcal{H}_{a}-\Sigma \varepsilon_{a b} \mathcal{H}^{b} \\
& -\left(\frac{1}{3} \theta+\frac{1}{4} \Sigma\right) Q_{a}+\frac{3}{2} \Omega \varepsilon_{a b} Q^{b}+\frac{1}{2} \Sigma_{a b} Q^{b}-\zeta_{a b}\left(\mathcal{E}^{b}+\frac{1}{2} \Pi^{b}\right)+\left(\mathcal{E}_{a b}+\frac{1}{2} \Pi_{a b}\right) a^{b}+3 \mathcal{H}_{a b} \Omega^{b}
\end{aligned}
$$

Magnetic Weyl divergence $\left(C_{5}\right)^{a} n_{a}$ :

$$
\begin{aligned}
\hat{\mathcal{H}}= & -\delta_{a} \mathcal{H}^{a}-\frac{1}{2} \varepsilon_{a b} \delta^{a} Q^{b}-\frac{3}{2} \phi \mathcal{H}-\left(3 \mathcal{E}+\mu+p-\frac{1}{2} \Pi\right) \Omega-Q \xi \\
& +2 \mathcal{H}_{a} a^{a}-3 \Omega_{a}\left(\mathcal{E}^{a}-\frac{1}{6} \Pi^{a}\right)+\zeta_{a b} \mathcal{H}^{a b}-\varepsilon_{a b} \Sigma^{a}{ }_{c}\left(\mathcal{E}^{b c}+\frac{1}{2} \Pi^{b c}\right)
\end{aligned}
$$

$\left(C_{5}\right)_{\bar{a}}$ :

$$
\begin{aligned}
\hat{\mathcal{H}}_{\bar{a}}-\frac{1}{2} \varepsilon_{a b} \hat{Q}^{b}= & \frac{1}{2} \delta_{a} \mathcal{H}-\delta^{b} \mathcal{H}_{a b}-\frac{1}{2} \varepsilon_{a b} \delta^{b} Q-\frac{3}{2}\left(\mathcal{E}+\frac{1}{2} \Pi\right) \varepsilon_{a b} \Sigma^{b}-\left(-\frac{3}{2} \mathcal{E}+\mu+p+\frac{1}{4} \Pi\right) \Omega_{a} \\
& -\frac{3}{2} \mathcal{H} a_{a}+\frac{1}{2} Q \varepsilon_{a b} a^{b}-3 \Omega \mathcal{E}_{a}+\frac{3}{2} \Sigma \varepsilon_{a b} \mathcal{E}^{b}-\frac{3}{2} \phi \mathcal{H}_{a}+\xi \varepsilon_{a b} \mathcal{H}^{b} \\
& -\frac{1}{2} \xi Q_{a}+\frac{1}{4} \phi \varepsilon_{a b} Q^{b}+\frac{1}{2} \Omega \Pi_{a}+\frac{3}{4} \Sigma \varepsilon_{a b} \Pi^{b} \\
& +\mathcal{H}_{a b} a^{b}-\zeta_{a b} \mathcal{H}^{b}-3\left(\mathcal{E}_{a b}-\frac{1}{6} \Pi_{a b}\right) \Omega^{b}+\frac{1}{2} \varepsilon_{a b} \zeta^{b c} Q_{c}
\end{aligned}
$$

\section{Constraint}

$\varepsilon^{a b} u^{c} R_{a b c}$

$$
\delta_{a} \Omega^{a}+\varepsilon_{a b} \delta^{a} \Sigma^{b}=(2 \mathcal{A}-\phi) \Omega-3 \xi \Sigma+\varepsilon_{a b} \zeta^{a c} \Sigma^{b}{ }_{c}+\mathcal{H}
$$

$N^{b c} R_{\bar{a} b c}:$

$$
\begin{aligned}
\frac{1}{2} \delta_{a} \phi-\varepsilon_{a b} \delta^{b} \xi-\delta^{b} \zeta_{a b}= & -2 \xi \varepsilon_{a b} a^{b}-\Omega\left(\Omega_{a}+\varepsilon_{a b} \Sigma^{b}-2 \varepsilon_{a b} \alpha^{b}\right)-\left(\frac{1}{3} \theta-\frac{1}{2} \Sigma\right)\left(\Sigma_{a}-\varepsilon_{a b} \Omega^{b}\right) \\
& -\left(\Sigma^{b}-\varepsilon^{b c} \Omega_{c}\right) \Sigma_{a b}-\frac{1}{2} \Pi_{a}-\mathcal{E}_{a}
\end{aligned}
$$

From $\left(C_{3}\right)_{a b} n^{b}$ and $\left(C_{1}\right)_{\bar{a}}$, or $n^{a} u^{c} R_{a \bar{b} c}$

$$
\begin{aligned}
\delta_{a} \Sigma-\frac{2}{3} \delta_{a} \theta+2 \varepsilon_{a b} \delta^{b} \Omega+2 \delta^{b} \Sigma_{a b}= & -\phi\left(\Sigma_{a}-\varepsilon_{a b} \Omega^{b}\right)-2 \xi\left(\Omega_{a}-3 \varepsilon_{a b} \Sigma^{b}\right)-4 \Omega \varepsilon_{a b} \mathcal{A}^{b} \\
& +2 \zeta_{a b} \Sigma^{b}+2 \varepsilon_{a b} \zeta^{b c} \Omega_{c}+\Sigma_{a b} a^{b}-2 \varepsilon_{a b} \mathcal{H}^{b}-Q_{a}
\end{aligned}
$$

Finally, we note that the equation formed from $\left(C_{3}\right)_{a b} n^{a} n^{b}$ is equivalent to Eqs (103) and (79).

It is worth noting that one of Eqs. (54), (55), (57) is redundant since (54) $=\frac{1}{3}(55)-(57)$. Also, note that there are no evolution equations for $\mathcal{A}, \mathcal{A}_{a}, \alpha_{a}$, and there is no propagation equation for $a_{a}$; these all must be determined by specifying a choice of frame. 


\section{E. Maxwell's Equations}

For completeness we also give the decomposition of Maxwell's equations, previously given in [10]. We decompose the electric and magnetic field vectors as

$$
\begin{aligned}
& E^{a}=\mathscr{E} n^{a}+\mathscr{E}^{a}, \\
& B^{a}=\mathscr{B} n^{a}+\mathscr{B}^{a},
\end{aligned}
$$

while the 3-current may be written as

$$
j^{a}=\mathcal{J} n^{a}+\mathcal{J}^{a} .
$$

Maxwell's equations then become:

$$
\begin{aligned}
\hat{\mathscr{E}}+\delta_{a} \mathscr{E}^{a}= & -\phi \mathscr{E}+\mathscr{E}_{a} a^{a}+2 \Omega \mathscr{B}+2 \Omega^{a} \mathscr{B}_{a}+\mu_{0} \rho_{\mathrm{e}}, \\
\hat{\mathscr{B}}+\delta_{a} \mathscr{B}^{a}= & -\phi \mathscr{B}+\mathscr{B}_{a} a^{a}-2 \Omega \mathscr{E}-2 \Omega^{a} \mathscr{E}_{a} \\
\dot{\mathscr{E}}-\varepsilon_{a b} \delta^{a} \mathscr{B}^{b}= & 2 \xi \mathscr{B}+\mathscr{E}^{a} \alpha_{a}-\left(\frac{2}{3} \theta-\Sigma\right) \mathscr{E}+\Sigma^{a} \mathscr{E}_{a}+\varepsilon_{a b}\left(\mathcal{A}^{a} \mathscr{B}^{b}+\Omega^{a} \mathscr{E}^{b}\right)-\mu_{0} \mathcal{J}, \\
\dot{\mathscr{B}}+\varepsilon_{a b} \delta^{a} \mathscr{E}^{b}= & -2 \xi \mathscr{E}+\mathscr{B}^{a} \alpha_{a}-\left(\frac{2}{3} \theta-\Sigma\right) \mathscr{B}+\Sigma^{a} \mathscr{B}_{a}-\varepsilon_{a b}\left(\mathcal{A}^{a} \mathscr{E}^{b}-\Omega^{a} \mathscr{B}^{b}\right), \\
\dot{\mathscr{E}}_{\bar{a}}+\varepsilon_{a b}\left(\hat{\mathscr{B}}^{b}-\delta^{b} \mathscr{B}\right)= & \xi \mathscr{B}_{a}-\left(\frac{1}{2} \phi+\mathcal{A}\right) \varepsilon_{a b} \mathscr{B}^{b}-\left(\frac{2}{3} \theta+\frac{1}{2} \Sigma\right) \mathscr{E}_{a}-\Omega \varepsilon_{a b} \mathscr{E}^{b} \\
& +\mathscr{E}^{b}\left(-\alpha_{a}+\Sigma_{a}+\varepsilon_{a b} \Omega^{b}\right)+\mathscr{B}_{a b}\left(\mathcal{A}^{b}-a^{b}\right)+\Sigma_{a b} \mathscr{E}^{b}-\varepsilon_{a b} \zeta^{b c} \mathscr{B}_{c}-\mu_{0} \mathcal{J}_{a}, \\
\dot{\mathscr{B}}_{\bar{a}}-\varepsilon_{a b}\left(\hat{\mathscr{E}}^{b}-\delta^{b} \mathscr{E}\right)= & -\xi \mathscr{E}_{a}+\left(\frac{1}{2} \phi+\mathcal{A}\right) \varepsilon_{a b} \mathscr{E}^{b}-\left(\frac{2}{3} \theta+\frac{1}{2} \Sigma\right) \mathscr{B}_{a}-\Omega \varepsilon_{a b} \mathscr{B}^{b} \\
& +\mathscr{B}\left(-\alpha_{a}+\Sigma_{a}+\varepsilon_{a b} \Omega^{b}\right)-\mathscr{E} \varepsilon_{a b}\left(\mathcal{A}^{b}-a^{b}\right)+\Sigma_{a b} \mathscr{B}^{b}+\varepsilon_{a b} \zeta^{b c} \mathscr{E}_{c} .
\end{aligned}
$$

Here, MKS units are used $\left(\mu_{0}\right)$, and $\rho_{e}$ is the charge density. The first two equations arise from the constraint ME, while the rest are the evolution ME. In flat space in the absence of currents and charges the rhs of these equations vanish (for a static 'natural' choice of frame). Thus, gravity modifies ME in the form of generalised currents. Note how the rotation terms $\xi, \Omega$ and $\Omega^{a}$ flip the parities of the EM fields.

\section{PERTURBATIONS OF SPHERICALLY SYMMETRIC AND LRS SPACETIMES}

The utility of the approach presented here is that for LRS spactimes, for which all quantities are rotationally symmetric about a preferred spatial direction (i.e., they admit a one-dimensional isotropy group), all the non-zero $1+1+2$ variables are scalars. This direction may be specified, for example, by a non-degenerate eigenvector of the electric Weyl tensor, or by the vorticity vector. A full discussion of LRS spacetimes in the covariant approach is given in [7]; see their Table 1 for a summary of the different cases which can occur, in a notation similar to that presented here.

The fact that background quantities are scalars in LRS spacetimes means that under linear perturbations, all vector and tensor quantities are automatically gauge invariant, by the Stewart-Walker Lemma [17]. We shall now give an overview of how to set up the perturbation equations.

In the background, which we shall take as a general LRS spacetime, all vector and tensor equations are automatically zero, resulting in the set

$$
\begin{aligned}
\dot{\phi}= & \left(\frac{2}{3} \theta-\Sigma\right)\left(\mathcal{A}-\frac{1}{2} \phi\right)+2 \xi \Omega+Q, \\
\dot{\xi}= & \left(\frac{1}{2} \Sigma-\frac{1}{3} \theta\right) \xi+\left(\mathcal{A}-\frac{1}{2} \phi\right) \Omega+\frac{1}{2} \mathcal{H}, \\
\dot{\Omega}= & +\mathcal{A} \xi+\Omega\left(\Sigma-\frac{2}{3} \theta\right) \\
\hat{\mathcal{A}}-\dot{\theta}= & -(\mathcal{A}+\phi) \mathcal{A}+\frac{1}{3} \theta^{2}+\frac{3}{2} \Sigma^{2}-2 \Omega^{2}+\frac{1}{2}(\mu+3 p)-\Lambda, \\
\dot{\Sigma}-\frac{2}{3} \hat{\mathcal{A}}= & \frac{1}{3}(2 \mathcal{A}-\phi) \mathcal{A}-\left(\frac{2}{3} \theta+\frac{1}{2} \Sigma\right) \Sigma-\frac{2}{3} \Omega^{2}-\mathcal{E}+\frac{1}{2} \Pi, \\
\dot{\mu}+\hat{Q}= & -\theta(\mu+p)-(\phi+2 \mathcal{A}) Q-\frac{3}{2} \Sigma \Pi, \\
\dot{Q}+\hat{p}+\hat{\Pi}= & -\left(\frac{3}{2} \phi+\mathcal{A}\right) \Pi-\left(\frac{4}{3} \theta+\Sigma\right) Q-(\mu+p) \mathcal{A}, \\
\dot{\mathcal{E}}+\frac{1}{2} \dot{\Pi}+\frac{1}{3} \hat{Q}= & +\left(\frac{3}{2} \Sigma-\theta\right) \mathcal{E}-\frac{1}{2}\left(\frac{1}{3} \theta+\frac{1}{2} \Sigma\right) \Pi \\
& +\frac{1}{3}\left(\frac{1}{2} \phi-2 \mathcal{A}\right) Q+3 \xi \mathcal{H}-\frac{1}{2}(\mu+p) \Sigma,
\end{aligned}
$$




$$
\begin{aligned}
\dot{\mathcal{H}} & =-3 \xi \mathcal{E}+\left(\theta+\frac{3}{2} \Sigma\right) \mathcal{H}+\Omega Q+\frac{3}{2} \xi \Pi, \\
\hat{\phi} & =-\frac{1}{2} \phi^{2}+2 \xi^{2}+\left(\frac{1}{3} \theta+\Sigma\right)\left(\frac{2}{3} \theta-\Sigma\right)-\frac{2}{3}(\mu+\Lambda)-\frac{1}{2} \Pi-\mathcal{E}, \\
\hat{\xi} & =-\phi \xi+\left(\frac{1}{3} \theta+\Sigma\right) \Omega, \\
\hat{\Sigma}-\frac{2}{3} \hat{\theta} & =-\frac{3}{2} \phi \Sigma-2 \xi \Omega-Q, \\
\hat{\Omega} & =+(\mathcal{A}-\phi) \Omega, \\
\hat{\mathcal{E}}-\frac{1}{3} \hat{\mu}+\frac{1}{2} \hat{\Pi} & =-\frac{3}{2} \phi\left(\mathcal{E}+\frac{1}{2} \Pi\right)+\left(\frac{1}{2} \Sigma-\frac{1}{3} \theta\right) Q+3 \Omega \mathcal{H}, \\
\hat{\mathcal{H}} & =-\frac{3}{2} \phi \mathcal{H}-\left(3 \mathcal{E}+\mu+p-\frac{1}{2} \Pi\right) \Omega-Q \xi, \\
0 & =(2 \mathcal{A}-\phi) \Omega-3 \xi \Sigma+\mathcal{H} .
\end{aligned}
$$

These equations were first presented in this form in [12] for LRS Class II models (which satisfy $\xi=\Omega=0 \Rightarrow \mathcal{H}=0$, and were shown to be consistent with the commutation relation (34).

It is perhaps easier to think of these in matrix form. Let

$$
\boldsymbol{X}=\left(\begin{array}{c}
\phi \\
\theta \\
\Sigma \\
\mathcal{A} \\
\Omega \\
\xi \\
\mathcal{E} \\
\mathcal{H} \\
\mu \\
p \\
Q \\
\Pi
\end{array}\right)
$$

be the column matrix of all non-zero scalar quantities. Depending on the LRS model in question $\boldsymbol{X}$ will not be this big. For example, for the Schwarzschild solution we have just $\boldsymbol{X}=(\phi, \mathcal{A}, \mathcal{E})^{T}$. Then, in general, this system of equations may be cast in the form

$$
\boldsymbol{\alpha} \dot{\boldsymbol{X}}+\boldsymbol{\beta} \hat{\boldsymbol{X}}=\boldsymbol{\Gamma} \boldsymbol{X}+\boldsymbol{X}^{T} \boldsymbol{\Delta} \boldsymbol{X}
$$

where the constant matrices $\boldsymbol{\alpha}, \boldsymbol{\beta}, \boldsymbol{\Gamma}, \boldsymbol{\Delta}$ may be read off from the above equations.

We can now set up the perturbative proceedure schematically as follows:

1. Find a complete set of gauge-invariant perturbation variables. This may be achieved by defining

$$
\boldsymbol{\Psi}_{a}=\delta_{a} \boldsymbol{X}
$$

i.e., by taking angular derivatives of the background variables we find a new set of gauge-invariant variables. The remaining GI variables are all the $1+1+2$ vectors and tensors: $\chi_{a}=\left(\mathcal{E}_{a}, a_{a}, \ldots\right), \boldsymbol{\chi}_{a b}=\left(\zeta_{a b}, \mathcal{E}_{a b}, \mathcal{H}_{a b}, \ldots\right)$, which obey linearised versions of the above $1+1+2$ equations. Under perturbations Eq. (108) becomes

$$
\boldsymbol{\alpha} \dot{\boldsymbol{X}}+\boldsymbol{\beta} \hat{\boldsymbol{X}}=\boldsymbol{\Gamma} \boldsymbol{X}+\boldsymbol{X}^{T} \boldsymbol{\Delta} \boldsymbol{X}+\boldsymbol{A} \delta^{a} \boldsymbol{\chi}_{a}+\boldsymbol{B} \varepsilon_{a b} \delta^{a} \chi^{b},
$$

where the matrices $\boldsymbol{A}, \boldsymbol{B}$ have constant coefficients. Evolution and propagation equations for the new GI variables $\boldsymbol{\Psi}_{a}$ may be found by taking the angular derivative of Eq. (110), and using the commutation relations (35) and (36), giving:

$$
\begin{aligned}
\boldsymbol{\alpha} \dot{\boldsymbol{\Psi}}_{a}+\boldsymbol{\beta} \hat{\mathbf{\Psi}}_{a}= & {\left[\boldsymbol{\Gamma}+\left(\frac{1}{2} \Sigma-\frac{1}{3} \theta-\frac{1}{2} \phi\right) \boldsymbol{\alpha}\right] \boldsymbol{\Psi}_{a}-(\Omega \boldsymbol{\alpha}+\xi \boldsymbol{\beta}) \varepsilon_{a b} \boldsymbol{\Psi}^{b} } \\
& +\boldsymbol{X}^{T} \boldsymbol{\Delta} \boldsymbol{\Psi}_{a}++\boldsymbol{\Psi}_{a}^{T} \boldsymbol{\Delta} \boldsymbol{X}+\boldsymbol{A} \delta_{a} \delta^{b} \boldsymbol{\chi}_{b}+\boldsymbol{B} \varepsilon_{b c} \delta_{a} \delta^{b} \boldsymbol{\chi}^{c} .
\end{aligned}
$$

These equations replace the corresponding system (110) in the $1+1+2$ equations.

2. Harmonic analysis Two parities of harmonics may be introduced, generalising the axial and polar modes for spherical symmetry. These were first defined in [8, 12], and are discussed in Appendix B. These are analogous to the scalar-vector-tensor decomposition in FLRW models. After this, all variables become scalars, which are functions of two affine parameters associated with $u^{a}$ and $n^{a}$. 
3. Master Variables At this stage the governing system of equations is linear in the perturbation variables $\mathbf{\Phi}$ and $\overline{\boldsymbol{\Phi}}$, which are the column vectors containing all the even and odd harmonically decomposed variables, and splits into two parities. We then have two linear systems of equations looking like

$$
\gamma \dot{\boldsymbol{\Phi}}+\lambda \hat{\boldsymbol{\Phi}}=\boldsymbol{\Xi \Phi}
$$

where $\boldsymbol{\Xi}$ is a matrix with coefficients depending only on the background parameters (as well as the harmonic index $k$ ), and $\boldsymbol{\gamma}, \boldsymbol{\lambda}$ are constant matrices. The true degrees of freedom of this system will be governed by a reduced set of frame independent master variables, which will obey a closed set of wave equations. Finding these can be tricky. All other variables are related to the master variables by quadrature, plus frame degrees of freedom. See [8] for the full details in the Schwarzschild case.

These are the key steps required given a particular LRS model is chosen. Steps 1 and 2 are algorithmic; step 3 can be very difficult.

\section{SUMMARY}

We have presented a new semi-tetrad approach to analysing Einstein's field equations. By introducing a single space-like vector into the $1+3$ approach we decomposed the $1+3$ equations into a system of evolution, propagation and constraint equations. These were supplemented by a $1+1+2$ decomposition of the Ricci equations for the spatial vector. Although presented in restricted form elsewhere, the full system was presented here for the first time.

A key feature of the approach is that under a complete decomposition all objects are covariantly defined scalars, 2-vectors in the sheet and transverse-traceless 2-tensors, also in the sheet. In an LRS spacetime, provided the spatial vector is chosen appropriately, all the vectors and tensors vanish, leaving just scalars. Under perturbations all indexed objects are first-order ensuring that there are no tensorial products; this ensures that we can introduce natural harmonic functions on the background which remove all tensorial properties of the equations. Finally, we are left with a system of gauge-invariant and covariant first-order PDEs to manipulate. The solution of this system provides the solution of the perturbation problem.

\section{Acknowledgments}

The author wishes to thank Peter Dunsby, George Ellis, Roy Maartens, and Mattias Marklund, for many useful discussions (though some time ago now...), and in particular wishes to thank Richard Barrett and Gerold Betschart for the same, as well as checking many of the equations, and GB for supplying Eqs. (38) - (41). CC is funded by the NRF (South Africa).

\section{APPENDIX A: USEFUL RELATIONS FOR DECOMPOSING EQUATIONS}

Given any $1+3$ vectors and tensors, we may decompose them as

$$
\begin{aligned}
x^{a} & =X n^{a}+X^{a}, \\
y^{a} & =Y n^{a}+Y^{a} \\
\psi_{a b} & =\psi_{\langle a b\rangle}=\Psi\left(n_{a} n_{b}-\frac{1}{2} N_{a b}\right)+2 \Psi_{(a} n_{b)}+\Psi_{a b}, \\
\phi_{a b} & =\phi_{\langle a b\rangle}=\Phi\left(n_{a} n_{b}-\frac{1}{2} N_{a b}\right)+2 \Phi_{(a} n_{b)}+\Phi_{a b} .
\end{aligned}
$$

Then we have the following expansions from $1+3$ quantities $\longrightarrow 1+1+2$ variables:

$$
\begin{aligned}
x_{a} x^{a} & =X^{2}+X_{a} X^{a} \\
\eta_{a b c} x^{b} y^{c} & =\left(\varepsilon_{b c} X^{b} Y^{c}\right) n_{a}+\varepsilon_{a b}\left(Y X^{b}-X Y^{b}\right) \\
x_{\langle a} y_{b\rangle} & =\frac{1}{3}\left(2 X Y-X_{c} Y^{c}\right)\left(n_{a} n_{b}-\frac{1}{2} N_{a b}\right)+\left[X Y_{(a}+Y X_{(a}\right] n_{b)}+X_{\{a} Y_{b\}}, \\
\psi_{a b} x^{b} & =\left(X \Psi+X_{b} \Psi^{b}\right) n_{a}-\frac{1}{2} \Psi X_{a}+X \Psi_{a}+\Psi_{a b} X^{b}, \\
\eta_{c d\langle a} x^{c} \psi_{b\rangle}{ }^{d} & =\varepsilon_{c d} X^{c} \Psi^{d}\left(n_{a} n_{b}-\frac{1}{2} N_{a b}\right)+\left[\left(X \Psi^{c}-\frac{3}{2} \Psi X^{c}\right) \varepsilon_{c(a}+\varepsilon_{c d} X^{c} \Psi_{(a}^{d}\right] n_{b)}+X \varepsilon_{c\{a} \Psi_{b\}}{ }^{c}-X^{c} \varepsilon_{c\{a} \Psi_{b\}}, \\
\psi_{a b} \psi^{a b} & =\frac{3}{2} \Psi^{2}+2 \Psi_{a} \Psi^{a}+\Psi_{a b} \Psi^{a b},
\end{aligned}
$$




$$
\begin{aligned}
\psi_{c\langle a} \phi_{b\rangle}{ }^{c}= & \left(\frac{1}{2} \Psi \Phi+\frac{1}{3} \Psi_{c} \Phi^{c}-\frac{1}{3} \Psi_{c d} \Phi^{c d}\right)\left(n_{a} n_{b}-\frac{1}{2} N_{a b}\right)+\left[\frac{1}{2} \Psi \Phi_{(a}+\frac{1}{2} \Phi \Psi_{(a}+\Psi^{c} \Phi_{c(a}+\Phi^{c} \Psi_{c(a}\right] n_{b)} \\
& -\frac{1}{2} \Psi \Phi_{a b}-\frac{1}{2} \Phi \Psi_{a b}+\Psi_{\{a} \Phi_{b\}}+\Psi_{c\{a} \Phi_{b\}}{ }^{c}, \\
\eta_{a b c} \psi_{d}^{b} \phi^{d c}= & n_{a} \varepsilon_{b c} \Psi_{d}^{b} \Phi^{d c}+\frac{3}{2} \varepsilon_{a b}\left(\Phi \Psi^{b}-\Psi \Phi^{b}\right) .
\end{aligned}
$$

For $1+3$ derivatives we find:

$$
\begin{aligned}
\dot{x}_{\langle a\rangle}= & \left(\dot{X}-X_{b} \alpha^{b}\right) n_{a}+X \alpha_{a}+\dot{X}_{\bar{a}}, \\
\dot{\psi}_{\langle a b\rangle}= & \left(\dot{\Psi}-2 \Psi_{c} \alpha^{c}\right) n_{a} n_{b}-\frac{1}{2} \dot{\Psi} N_{a b}+\left[3 \Psi \alpha_{(a}+2 \dot{\Psi}_{(\bar{a}}-2 \alpha^{c} \Psi_{c(a}\right] n_{b)}+2 \Psi_{(a} \alpha_{b)}+\dot{\Psi}_{\{a b\}}, \\
\mathrm{D}_{a} x^{a}= & \hat{X}+X \phi-X_{a} a^{a}+\delta_{a} X^{a}, \\
\eta_{a b c} \mathrm{D}^{b} x^{c}= & \left(2 X \xi+\varepsilon_{b c} \delta^{b} X^{c}\right) n_{a}+\xi X_{a}+\varepsilon_{a b}\left[-X a^{b}+\delta^{b} X-\hat{X}^{b}-\frac{1}{2} \phi X^{b}-\zeta^{b c} X_{c}\right], \\
\mathrm{D}_{\langle a} x_{b\rangle}= & \frac{1}{3}\left[2 \hat{X}-\phi X-2 X_{c} a^{c}-\delta_{c} X^{c}\right]\left(n_{a} n_{b}-\frac{1}{2} N_{a b}\right) \\
& +\left[X a_{(a}+\delta_{(a} X+\hat{X}_{(\bar{a}}-\frac{1}{2} \phi X_{(a}+X^{c}\left(\xi \varepsilon_{c(a}-\zeta_{c(a}\right)\right] n_{b)}+X \zeta_{a b}+\delta_{\{a} X_{b\}}, \\
\mathrm{D}^{b} \psi_{a b}= & \left(\hat{\Psi}+\frac{3}{2} \phi \Psi-2 \Psi_{b} a^{b}+\delta_{b} \Psi^{b}-\Psi_{b c} \zeta^{b c}\right) n_{a}+\hat{\Psi}_{\bar{a}}+\frac{3}{2} \phi \Psi_{a}+\frac{3}{2} \Psi a_{a}-\frac{1}{2} \delta_{a} \Psi \\
& -\Psi_{a b} a^{b}+\left[-\xi \varepsilon_{a b}+\zeta_{a b}\right] \Psi^{b}+\delta^{b} \Psi_{a b}, \\
\eta_{c d\langle a} \mathrm{D}^{c} \psi_{b\rangle}{ }^{d}= & \left(3 \xi \Psi+\varepsilon_{c d} \delta^{c} \Psi^{d}-\varepsilon_{c d} \Psi^{d e} \zeta_{e}^{c}\right)\left(n_{a} n_{b}-\frac{1}{2} N_{a b}\right) \\
& +\left\{\left[-\frac{3}{2} \delta^{c} \Psi+\frac{3}{2} \Psi a^{c}+\hat{\Psi}^{c}+\frac{1}{2} \phi \Psi^{c}+2 \Psi_{d} \zeta^{c d}\right] \varepsilon_{c(a}+5 \xi \Psi_{(a}+\varepsilon^{c d}\left[\Psi_{d} \zeta_{c(a}+\delta_{c} \Psi_{d(a}\right]\right\} n_{b)} \\
& -\varepsilon_{c\{a} \delta^{c} \Psi_{a\}}+2 \varepsilon_{c\{a} a^{c} \Psi_{b\}}+\varepsilon_{c\{a} \hat{\Psi}_{b\}}^{c}+\frac{1}{2} \phi \varepsilon_{c\{a} \Psi_{b\}}^{c}-\frac{3}{2} \Psi \varepsilon_{c\{a} \zeta_{b\}}^{c}+\xi \Psi_{a b}+\varepsilon_{c\{a} \Psi_{b\} d} \zeta^{c d} .
\end{aligned}
$$

Given any relation in $1+3$ notation, these relations may be substituted directly to aid decomposition.

\section{APPENDIX B: HARMONIC FUNCTIONS}

We introduce dimensionless harmonic functions $Q$, defined on any LRS background, as eigenfunctions of the 2dimensional Laplace-Beltrami operator:

$$
\delta^{2} Q=-\frac{k^{2}}{r^{2}} Q, \quad \hat{Q}=0=\dot{Q} \quad\left(0 \leq k^{2}\right) .
$$

The function $r$ is, up to an irrelevant constant, covariantly defined by

$$
\frac{\hat{r}}{r} \equiv \frac{1}{2} \phi, \quad \frac{\dot{r}}{r} \equiv \frac{1}{3} \theta-\frac{1}{2} \Sigma, \quad \delta_{a} r \equiv 0 .
$$

While we haven't chosen a specific basis for $Q$, we can now expand any first order scalar $\psi$ in terms of these functions schematically as

$$
\psi=\sum_{k} \psi_{\mathrm{s}}^{(k)} Q^{(k)}=\psi_{\mathrm{s}} Q
$$

where the sum (or integral) over $k$ is implicit in the last equality. The $\mathbf{S}$ subscript reminds us that $\psi$ is a scalar, and that a harmonic expansion has been made.

We also need to expand vectors in harmonics. We therefore define the even (electric) parity vector harmonics as

$$
Q_{a}^{(k)}=r \delta_{a} Q^{(k)} \quad \Rightarrow \quad \hat{Q}_{\bar{a}}=0=\dot{Q}_{\bar{a}}, \quad \delta^{2} Q_{a}=\left(1-k^{2}\right) r^{-2} Q_{a} ;
$$

where the $(k)$ superscript is implicit, and we define odd (magnetic) parity vector harmonics as

$$
\bar{Q}_{a}^{(k)}=r \varepsilon_{a b} \delta^{b} Q^{(k)} \quad \Rightarrow \quad \hat{\bar{Q}}_{\bar{a}}=0=\dot{\bar{Q}}_{\bar{a}}, \quad \delta^{2} \bar{Q}_{a}=\left(1-k^{2}\right) r^{-2} \bar{Q}_{a} .
$$

Note that $\bar{Q}_{a}=\varepsilon_{a b} Q^{b} \Leftrightarrow Q_{a}=-\varepsilon_{a b} \bar{Q}^{b}$, so that $\varepsilon_{a b}$ is a parity operator. The crucial difference between these two types of vector harmonics is that $\bar{Q}_{a}$ is solenoidal, so

$$
\delta^{a} \bar{Q}_{a}=0
$$


while

$$
\delta^{a} Q_{a}=-k^{2} r^{-1} Q
$$

Note also that

$$
\varepsilon_{a b} \delta^{a} Q^{b}=0, \quad \text { and } \quad \varepsilon_{a b} \delta^{a} \bar{Q}^{b}=+k^{2} r^{-1} Q .
$$

The harmonics are orthogonal: $Q^{a} \bar{Q}_{a}=0$ (for each $k$ ), which implies that any first-order vector $\psi_{a}$ can now be written

$$
\psi_{a}=\sum_{k} \psi_{\vee}^{(k)} Q_{a}^{(k)}+\bar{\psi}_{\vee}^{(k)} \bar{Q}_{a}^{(k)}=\psi_{\vee} Q_{a}+\bar{\psi}_{\vee} \bar{Q}_{a}
$$

Again, we implicitly assume a sum over $k$ in the last equality, and the $\mathrm{V}$ subscript reminds us that $\psi_{a}$ is a vector expanded in harmonics.

Similarly we define even and odd tensor spherical harmonics as

$$
\begin{aligned}
& Q_{a b}=r^{2} \delta_{\{a} \delta_{b\}} Q, \quad \Rightarrow \quad \hat{Q}_{a b}=0=\dot{Q}_{a b}, \\
& \bar{Q}_{a b}=r^{2} \varepsilon_{c\{a} \delta^{c} \delta_{b\}} Q, \quad \Rightarrow \quad \hat{\bar{Q}}_{a b}=0=\dot{\bar{Q}}_{a b},
\end{aligned}
$$

which are orthogonal: $Q_{a b} \bar{Q}^{a b}=0$, and are parity inversions of one another: $Q_{a b}=-\varepsilon_{c\{a} \bar{Q}_{b\}}{ }^{c} \Leftrightarrow \bar{Q}_{a b}=\varepsilon_{c\{a} Q_{b\}}{ }^{c}$. Any first-order tensor may be expanded

$$
\Psi_{a b}=\sum_{k} \Psi_{\top}^{(k)} Q_{a b}^{(k)}+\bar{\Psi}_{\top}^{(k)} \bar{Q}_{a b}^{(k)}=\Psi_{\mathrm{T}} Q_{a b}+\bar{\Psi}_{\mathrm{T}} \bar{Q}_{a b}
$$

[1] G. F. R. Ellis and H. van Elst, in M. Lachieze-Rey (ed.), Theoretical and Observational Cosmology, NATO Science Series, Kluwer Academic Publishers (1998) arXiv:gr-qc/9812046v4

[2] Ellis, G.F.R. and Bruni, M. Phys Rev. D 401804 (1989)

[3] Greenberg, P.J., J. Math. Anal. Applic. 30128 (1970)

[4] Tsamparlis, M. and Mason, D.P., J. Math. Phys. 241577 (1983)

[5] Mason, D.P. and Tsamparlis, M., J. Math. Phys. 262881 (1985)

[6] Tsamparlis, M. J. Math. Phys. 331472 (1992)

[7] van Elst, H. and Ellis, G. F. R. Class. Quantum Grav. 13 1099-1127 (1996)

[8] Clarkson, C. A. and Barrett, R. Class. Quantum Grav. 203855 (2003)

[9] Regge, T. and Wheeler, J.A. Phys. Rev. 1081063 (1957)

[10] Clarkson, C. A., Marklund, M., Betschart, G. and Dunsby, P. K. S. 2003 Astrophys.J. $613492-505$ (2004)

[11] Burston R. and Lun, A. arXiv:gr-qc/0611052 (2006)

[12] Betschart, G. and Clarkson, C. A., Class. Quantum Grav. 215587-5607 (2004)

[13] Burston R. and Lun, A. arXiv:gr-qc/0610062 (2006)

[14] Challinor, A.D. and Lasenby, A.N. Phys. Rev. D 58023001 (1998); Astrophys. J. 5131 (1999)

[15] Maartens, R., Gebbie, T. and Ellis, G.F.R. Phys. Rev. D 59083506 (1999)

[16] Zafiris, E., J. Math. Phys. 385854 (1997)

[17] Stewart, J.M. and Walker, M. Proc. R. Soc. London A 43149 (1974) 